$$
\begin{aligned}
&\left(\Phi, \Phi^{\prime}\right)= \sum_{k_{i}, k_{i}}\left[H\left(k_{i}\right), H\left(k_{i}^{\prime}\right)\right] \\
& \times \amalg\left(\frac{\left(\bar{\tau}_{i}\right)^{k_{i}}}{k_{i} !}\right) \Pi\left(\frac{\left(\tau_{i}^{\prime}\right)^{k^{\prime} i}}{k_{j}^{\prime} !}\right) \\
&=\int \exp \left[\Phi\left(\bar{\tau}_{i}, \bar{\zeta}\right)+\Phi\left(\tau_{j}^{\prime}, \zeta\right)\right] d \mu_{18}(\zeta) .
\end{aligned}
$$

The integration may first be performed with respect to $\zeta_{2}, \zeta_{4}, \zeta_{8}$, using Eq. (B5), and then $\zeta_{5}$, using Eq.
(B9). The result may then be expanded and integrated with respect to the remaining variables. The sums may be contracted by means of binomial identities. The following result is obtained:

$$
\begin{gathered}
\left(\Phi, \Phi^{\prime}\right)=\sum\left(\bar{\tau}_{0} \tau_{0}^{\prime}\right)^{k_{0}} \beta^{\alpha_{2}}(-d)^{\alpha_{2}}[(4)+(6)]^{\alpha_{2}}[(3)(4)]^{s_{3}} \\
\quad \times[(2)+(3)]^{z_{4}}[(5)(6)]^{z_{5}}[(1)+(5)]^{z_{*}} \cdot S, \quad(\mathrm{~B} 18)
\end{gathered}
$$

where $(i)=\bar{\tau}_{i} \tau_{i}^{\prime}$ (no summation) and $S$ is the factor

$$
\begin{aligned}
& S=\frac{\left(m_{1}+k_{0}+\alpha_{2}+1\right) !\left(2 m_{1}+2 k_{0}+2 \alpha_{2}+\alpha_{3}-\alpha_{1}+z_{4}+z_{6}+3\right) !}{k_{0} ! 2 z_{3} ! z_{4} ! z_{5} ! z_{6} ! \alpha_{1} !\left(m_{1}+k_{0}+\alpha_{2}-\alpha_{1}+1\right) ! \alpha_{2} ! \alpha_{3} !} \\
& m_{1}=z_{3}+z_{5}+\alpha_{1}+\alpha_{3} \text {, } \\
& d=(1)(3)+(2)(5)+(3)(5) \\
& =\left(\overline{\tau_{1} \tau_{3} \tau_{5}-\tau_{2} \tau_{4} \tau_{6}}\right)\left(\tau_{1}^{\prime} \tau_{3}^{\prime} \tau_{5}^{\prime}-\tau_{2}^{\prime} \tau_{4}^{\prime} \tau_{6}^{\prime}\right) \text {. }
\end{aligned}
$$$$
\times \frac{\left(2 m_{1}+k_{0}+2 \alpha_{2}-\alpha_{1}+z_{4}+z_{6}+2\right) !\left(m_{1}+k_{0}+\alpha_{2}-\alpha_{1}+z_{4}+z_{8}+1\right) !}{\left(2 m_{1}+2 k_{0}+2 \alpha_{2}-\alpha_{1}+z_{4}+z_{6}+3\right) !},
$$

$(0)^{k_{0}}(1)^{\rho_{1}} \cdots(6)^{\rho_{0}}\left(\overline{\left(\tau_{1} \tau_{3} \tau_{5}\right.}\right)^{x_{1}}\left(\overline{\tau_{2} \tau_{4} \tau_{6}}\right)^{x_{3}}\left(\tau_{1}^{\prime} \tau_{3}^{\prime} \tau_{5}^{\prime}\right)^{y_{1}}\left(\tau_{2}^{\prime} \tau_{4}^{\prime} \tau_{\theta}^{\prime}\right)^{y_{\mathbf{y}}}$

where

$$
x_{1}+x_{2}=N=y_{1}+y_{2}
$$

with the appropriate coefficient giving the result $\left[H\left(k_{i}\right), H\left(k_{i}^{\prime}\right)\right]$.
Expression (B18) must now be expanded out. The terms may be collected in the form:

\title{
Recoupling Coefficients for the Group SU(3)*
}

\author{
M. RESNIKOFF $\dagger$ \\ Harrison M. Randall Laboratory of Physics, University of Michigan, Ann Arbor, Michigan \\ (Received 21 February 1966)
}

\begin{abstract}
The Hilbert space method, employed in the previous article to obtain the coupling coefficients of $S U(3)$, is used here to obtain the recoupling, or $6(\lambda \mu)$, coefficients of $S U(3)$. The coefficients are formulated in terms of a generating function involving an integral, and an explicit expression is integrated out for the general nondegenerate case. The symmetries of the $6(\lambda \mu)$ coefficients are discussed.
\end{abstract}

\section{INTRODUCTION}

$\mathrm{T}$ HE $6(\lambda \mu)$ coefficient of $S U(3)$, which relates the alternate ways three representations $\left[\lambda_{i}, \mu_{i}\right]$, $i=1,2,3$, may be coupled, can be written in the form ${ }^{1}$

* Work supported in part by the National Science Foundation (Grant No. GP-1536). This paper is based on a dissertation submitted in partial fulfillment of the requirements for the degree of Doctor of Philosophy at the University of Michigan, Ann Arbor, Michigan.

$\dagger$ Present address: Department of Physics and Astronomy, University of Maryland, College Park, Maryland.

1 A form similar to this has been derived by J. J. de Swart, Nuovo Cimento 31, 420 (1964). Equation (1.1) is the recoupling coefficient multiplied by the factor

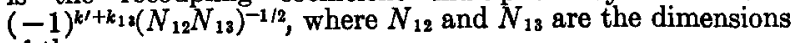
of the spaces

(see Ref. 3 below).

$$
\mathfrak{S}_{\lambda_{1}, \mu_{1},}, \mathfrak{S}_{\lambda_{18}, \mu_{1}} \text {, }
$$

$$
\begin{aligned}
& {\left[\begin{array}{ccc}
\lambda \mu ; k, k^{\prime} & \lambda_{2} \mu_{2} & \lambda_{12} \mu_{12} ; k_{12} \\
\lambda_{1} \mu_{1} & \lambda_{3} \mu_{3} & \lambda_{13} \mu_{13} ; k_{13}
\end{array}\right]} \\
& =\sum_{\alpha_{i}}\left\{\begin{array}{ccc}
\lambda_{1} \mu_{1} & \lambda_{2} \mu_{2} & \lambda_{12} \mu_{12} \\
\alpha_{1} & \alpha_{2} & \alpha_{12}
\end{array}\right\}_{k_{13}}\left\{\begin{array}{ccc}
\lambda_{12} \mu_{12} & \lambda_{3} \mu_{3} & \lambda_{\mu} \\
\alpha_{12} & \alpha_{3} & \alpha
\end{array}\right\}_{k} \\
& \quad \times\left\{\begin{array}{ccc}
\mu_{13} \lambda_{13} & \mu_{2} \lambda_{2} & \mu \lambda \\
-\alpha_{13} & -\alpha_{2} & -\alpha
\end{array}\right\}_{k^{\prime}}\left\{\begin{array}{ccc}
\mu_{1} \lambda_{1} & \mu_{3} \lambda_{3} \mu_{13} \lambda_{13} \\
-\alpha_{1} & -\alpha_{3} & -\alpha_{13}
\end{array}\right\}_{k_{13}},
\end{aligned}
$$

where use has been made of the orthogonal properties $^{2}$ and the symmetry properties of the $3(\lambda \mu)$ coefficients derived in the previous paper. ${ }^{3}$

J. J. de Swart, Rev. Mod. Phys. 35, 916 (1963).

${ }^{3}$ M. Resnikoff, preceding paper, J. Math. Phys. 8, 63 (1967). This article is hereafter referred to as (I). 


\section{SYMMETRY OF THE $\sigma\left(\lambda_{\mu}\right)$ COEFFICIENTS}

From the symmetries of the $3(\lambda \mu)$ coefficients ${ }^{4}$ [see Sec. 3D. of (I)], many symmetries of the $6(\lambda \mu)$ coefficients are apparent from Eq. (1.1).

\section{Exchange of columns 1, 2}

\section{Let}

$\lambda_{1} \mu_{1} \leftrightarrow \mu_{3} \lambda_{3}, \quad \lambda \mu \leftrightarrow \lambda_{2} \mu_{2}, \quad \lambda_{13} \mu_{13} \leftrightarrow \mu_{13} \lambda_{13}$

be exchanged and let $k \leftrightarrow k_{12}$ also be exchanged in Eq. (1.1). The right-hand side of Eq. (1.1) becomes

$$
\begin{aligned}
& \sum_{\alpha_{i}}\left\{\begin{array}{ccc}
\mu_{3} \lambda_{3} & \lambda \mu & \lambda_{12} \mu_{12} \\
-\alpha_{3} & \alpha & \alpha_{12}
\end{array}\right\}_{k}\left\{\begin{array}{ccc}
\lambda_{12} \mu_{12} & \mu_{1} \lambda_{1} & \lambda_{2} \mu_{2} \\
\alpha_{12} & -\alpha_{1} & \alpha_{2}
\end{array}\right\}_{k_{1},} \\
& \times\left\{\begin{array}{ccc}
\lambda_{13} \mu_{13} & \lambda \mu & \lambda_{2} \mu_{2} \\
\alpha_{13} & \alpha & \alpha_{2}
\end{array}\right\}_{k},\left\{\begin{array}{ccc}
\lambda_{3} \mu_{3} & \lambda_{1} \mu_{1} & \lambda_{13} \mu_{13} \\
\alpha_{3} & \alpha_{1} & \alpha_{13}
\end{array}\right\}_{k_{13}} .
\end{aligned}
$$

The right-hand side of Eq. (2.1b) is equal to the right-hand side of Eq. (1.1) [using Eqs. (3.56), (3.59), (3.61), (3.68) of (I)], except for a phase. The result follows that

$$
\begin{aligned}
& {\left[\begin{array}{ccc}
\lambda \mu ; k, k^{\prime} & \lambda_{2} \mu_{2} & \lambda_{12} \mu_{12} ; k_{12} \\
\lambda_{1} \mu_{1} & \lambda_{3} \mu_{3} & \lambda_{13} \mu_{13} ; k_{13}
\end{array}\right]} \\
& \quad=(-1)^{A}\left[\begin{array}{ccc}
\lambda_{2} \mu_{2} ; k_{12} k^{\prime} & \lambda_{\mu} & \lambda_{12} \mu_{12} ; k \\
\mu_{3} \lambda_{3} & \mu_{1} \lambda_{1} & \mu_{13} \lambda_{13} ; k_{13}
\end{array}\right],
\end{aligned}
$$

where

$$
\begin{aligned}
A=\lambda_{1}+\lambda_{3}+ & \lambda+\mu_{2} \\
& -\left(\mu+\mu_{1}+\mu_{3}+\lambda_{2}\right)+k+k_{12} .
\end{aligned}
$$

The other relations follow similarly.

\section{Exchange of columns 1, 3}

Let

$\lambda_{1} \mu_{1} \leftrightarrow \mu_{13} \lambda_{13}, \quad \lambda \mu \leftrightarrow \mu_{12} \lambda_{12}, \quad \lambda_{2} \mu_{2} \leftrightarrow \mu_{2} \lambda_{2}$,

and $k_{12} \leftrightarrow k^{\prime}$, be exchanged. Then

$$
\begin{aligned}
& {\left[\begin{array}{ccc}
\lambda \mu ; k, k^{\prime} & \lambda_{2} \mu_{2} & \lambda_{12} \mu_{12} ; k_{12} \\
\lambda_{1} \mu_{1} & \lambda_{3} \mu_{3} & \lambda_{13} \mu_{13} ; k_{13}
\end{array}\right]} \\
& \quad=(-1)^{B}\left[\begin{array}{ccc}
\mu_{12} \lambda_{12} ; k, k_{12} & \mu_{2} \lambda_{2} & \mu \lambda ; k^{\prime} \\
\mu_{13} \lambda_{13} & \lambda_{3} \mu_{3} & \mu_{1} \lambda_{1} ; k_{13}
\end{array}\right],
\end{aligned}
$$

where

$$
\begin{aligned}
B=\mu+\mu_{1}+ & \lambda_{2}+\lambda_{13} \\
- & \left(\lambda+\mu_{12}+\mu_{13}+\lambda_{1}\right)+k+k_{13} .
\end{aligned}
$$

These symmetries relate six of the $6(\lambda \mu)$ symbols.

4 J. R. Derome and W. T. Sharp, J. Math. Phys. 6, 1584 (1965), have discussed symmetries for the $6-j$ symbols of a general group. In contrast to their paper, the phase and the method of labeling degenerate states is specified here, and this leads to simpler relations. de Swart (Ref. 1) obtains symmetry relations for octet recouplings.

\section{Inversion of columns 1,2}

Let

$$
\lambda_{1} \mu_{1} \leftrightarrow \lambda \mu, \quad \lambda_{2} \mu_{2} \leftrightarrow \mu_{3} \lambda_{3},
$$

and $k_{12} \leftrightarrow k, k_{13} \leftrightarrow k^{\prime}$, be exchanged. Then

$$
\begin{aligned}
& {\left[\begin{array}{ccc}
\lambda \mu ; k, k^{\prime} & \lambda_{2} \mu_{2} & \lambda_{12} \mu_{12} ; k_{12} \\
\lambda_{1} \mu_{1} & \lambda_{3} \mu_{3} & \lambda_{13} \mu_{13} ; k_{13}
\end{array}\right]} \\
& =\left[\begin{array}{cc}
\lambda_{1} \mu_{1} ; k_{12}, k_{13} & \mu_{3} \lambda_{3} \lambda_{12} \mu_{12} ; k \\
\lambda \mu & \mu_{2} \lambda_{2} \lambda_{13} \mu_{13} ; k^{\prime}
\end{array}\right] .
\end{aligned}
$$

\section{Inversion of columns 1, 3}

Let

$$
\lambda \mu \leftrightarrow \mu_{1} \lambda_{1}, \quad \lambda_{12} \mu_{12} \leftrightarrow \mu_{13} \lambda_{13}
$$

and $k_{12} \leftrightarrow k^{\prime}, k_{13} \leftrightarrow k$, be exchanged. Then,

$$
\begin{aligned}
& {\left[\begin{array}{ccc}
\lambda \mu ; k, k^{\prime} & \lambda_{2} \mu_{2} & \lambda_{12} \mu_{12} ; k_{12} \\
\lambda_{1} \mu_{1} & \lambda_{3} \mu_{3} & \lambda_{13} \mu_{13} ; k_{13}
\end{array}\right]} \\
& =\left[\begin{array}{cc}
\mu_{1} \lambda_{1} ; k_{13}, k_{12} & \lambda_{2} \mu_{2} \mu_{13} \lambda_{13} ; k^{\prime} \\
\mu \lambda & \lambda_{3} \mu_{3} \mu_{12} \lambda_{12} ; k
\end{array}\right] .
\end{aligned}
$$

Finally, if the partition numbers are exchanged, $\lambda_{i} \leftrightarrow \mu_{i}$, then the right-hand side of Eq. (1.1) is a sum over conjugate $3(\lambda \mu)$ symbols, and the symmetry relation, Eq. (3.68) of (I), may be employed, with the result

$$
\begin{aligned}
& {\left[\begin{array}{cc}
\lambda \mu ; k, k^{\prime} & \lambda_{2} \mu_{2} \lambda_{12} \mu_{12} ; k_{12} \\
\lambda_{1} \mu_{1} & \lambda_{3} \mu_{3} \lambda_{13} \mu_{13} ; k_{13}
\end{array}\right]} \\
& \quad=(-1)^{c}\left[\begin{array}{cc}
\mu \lambda ; k, k^{\prime} & \mu_{2} \lambda_{2} \mu_{12} \lambda_{12} ; k_{12} \\
\mu_{1} \lambda_{1} & \mu_{3} \lambda_{3} \mu_{13} \lambda_{13} ; k_{13}
\end{array}\right]
\end{aligned}
$$

where

$$
C=k+k+k_{12}+k_{13} \text {. }
$$

This symmetry is present in $S U(3)$ because the base vector $|\lambda \mu ; \alpha\rangle$ and the conjugate base vector $|\lambda \mu ; \alpha\rangle_{c}$ are in different Hilbert spaces. In $S U(2), v_{m}^{i}$ and $w_{m}^{j}$ are members of the same Hilbert space. ${ }^{j}$ The $3-j$ symbol

$$
\left(\begin{array}{ccc}
j_{1} & j_{2} & j_{3} \\
m_{1} & m_{2} & m_{3}
\end{array}\right)
$$

and its conjugate

$$
\left(\begin{array}{ccc}
j_{1} & j_{2} & j_{3} \\
-m_{1} & -m_{2} & -m_{3}
\end{array}\right)
$$

are related by a phase, but since the spaces, labeled by $j$, are the same, a change to the conjugate $6-j$ symbol yields no further relations. In general, then, $486(\lambda \mu)$ coefficients are related by a phase.

\footnotetext{
b. Bargmann, Rev. Mod. Phys. 34, 829 (1962).
} 


\section{3. $6\left(\lambda_{u}\right)$ COEFFICIENT EXPRESSED AS AN INTEGRAL}

Notation: Let the variables of the base vector $|\lambda \mu ; \alpha\rangle$ be written $f\left(\zeta, \zeta^{\prime}\right)$ or $f(\zeta, \delta)$, and the base vector with complex conjugate variables $\bar{\zeta}, \bar{\delta}$ be written $\overline{|\lambda \mu ; \alpha\rangle}$. Also, let the invariants $h_{k}\left(\rho_{i}\right)$ be written $h_{k}\left(\zeta_{1}, \delta_{1} ; \zeta_{2}, \delta_{2} ; \zeta_{3}, \delta_{3}\right)$, where the explicit functional dependence is exhibited. ${ }^{6}$

The variables of the invariants $h_{k}\left(\rho_{i}\right)$ are chosen such that a product of four $h_{k}\left(\rho_{i}\right)$, integrated over the variables $\zeta_{i}$, yields a multiple of the $6(\lambda \mu)$ coefficient, as given by Eq. (1.1). First, associate, with each $3(\lambda \mu)$ coefficient appearing in Eq. (1.1) an appropriate invariant $h_{i}\left(\rho_{i}\right), j=1, \ldots, 4$ :

$$
\begin{aligned}
h_{1} \equiv & h_{k_{12}}\left(\zeta_{1}, \delta_{1} ; \zeta_{2}, \delta_{2} ; \zeta_{12}, \delta_{12}\right) \\
= & \sum_{\alpha_{1} \alpha_{2} \alpha_{1},}\left\{\begin{array}{ccc}
\lambda_{1} \mu_{1} & \lambda_{2} \mu_{2} & \lambda_{12} \mu_{12} \\
\alpha_{1} & \alpha_{2} & \alpha_{12}
\end{array}\right\}_{k_{12},} \\
& \times\left|\lambda_{1} \mu_{1} ; \alpha_{1}\right\rangle\left|\lambda_{2} \mu_{2} ; \alpha_{2}\right\rangle\left|\lambda_{12} \mu_{12} ; \alpha_{12}\right\rangle_{c} .
\end{aligned}
$$

In

$$
\begin{array}{r}
h_{k}\left(\zeta_{12}, \delta_{12} ; \zeta_{3}, \delta_{3} ; \zeta, \delta\right)=\sum_{\alpha_{12} \alpha^{\prime}, \alpha_{2}, \alpha}\left\{\begin{array}{ccc}
\lambda_{12} \mu_{12} & \lambda_{3} \mu_{3} & \lambda \mu \\
\alpha_{12}^{\prime} & \alpha_{3} & \alpha
\end{array}\right\}_{k} \\
\times\left|\lambda_{12} \mu_{12} ; \alpha_{12}^{\prime}\right\rangle\left|\lambda_{3} \mu_{3} ; \alpha_{3}\right\rangle|\lambda \mu ; \alpha\rangle_{c}
\end{array}
$$

exchange $\zeta_{12} \leftrightarrow \delta_{12}, \zeta \leftrightarrow \delta$, and complex conjugate these variables to get

$$
\begin{aligned}
h_{2} \equiv & h_{k}\left(\bar{\delta}_{12}, \bar{\zeta}_{12} ; \zeta_{3}, \delta_{3} ; \bar{\delta}, \bar{\zeta}\right) \\
= & \sum_{\alpha_{12} \alpha_{\alpha} \alpha}\left\{\begin{array}{ccc}
\lambda_{12} \mu_{12} & \lambda_{3} \mu_{3} & \lambda \mu \\
\alpha_{12}^{\prime} & \alpha_{3} & \alpha
\end{array}\right\}_{k}\left\{\frac{\left(\lambda_{12}+1\right) !(\mu+1) !}{\left(\mu_{12}+1\right) !(\lambda+1) !}\right\} \\
& \left.\times\left|\overline{\left.\lambda_{12} \mu_{12} ; \alpha_{12}\right\rangle_{c}}\right| \lambda_{3} \mu_{3} ; \alpha_{3}\right\rangle|\overline{\lambda \mu ; \alpha}\rangle .
\end{aligned}
$$

The degree conditions [see Sec. $3 \mathrm{C}$ of (I)] are chosen [Eq. (3.6)] such that the $3(\lambda \mu)$ coefficient appearing in Eq. (1.1) is obtained. Similarly,

$$
\begin{aligned}
& h_{3} \equiv h_{k^{\prime}} \cdot\left(\bar{\delta}_{13}, \bar{\zeta}_{13} ; \bar{\delta}_{2}, \bar{\zeta}_{2} ; \zeta, \delta\right) \\
&=\sum_{\alpha^{\prime} \alpha_{\xi^{\prime} \alpha^{\prime}, 1}}\left\{\begin{array}{lll}
\mu_{13} \lambda_{13} & \mu_{2} \lambda_{2} & \mu \lambda \\
-\alpha_{13}^{\prime} & -\alpha_{2}^{\prime} & -\alpha^{\prime}
\end{array}\right\}_{k^{\prime}} \\
& \times\left\{\frac{\left(\mu_{2}+1\right) !\left(\mu_{13}+1\right) !}{\left(\lambda_{2}+1\right) !\left(\lambda_{13}+1\right) !}\right. \\
& \times \overline{\left|\lambda_{13} \mu_{13} ; \alpha_{13}^{\prime}\right\rangle} \mid \frac{\left|\lambda_{2} \mu_{2} ; \alpha_{2}^{\prime}\right\rangle}{\left|\lambda \mu ; \alpha^{\prime}\right\rangle}
\end{aligned}
$$

and

$$
\begin{aligned}
& h_{4} \equiv h_{k_{12}}\left(\bar{\delta}_{1}, \bar{\zeta}_{1} ; \bar{\delta}_{3}, \bar{\zeta}_{3} ; \zeta_{13}, \delta_{13}\right) \\
& =\sum_{\alpha_{1} \alpha_{z^{\prime}} \alpha_{12} \cdot}\left\{\begin{array}{lll}
\mu_{1} \lambda_{1} & \mu_{3} \lambda_{3} & \mu_{13} \lambda_{13} \\
-\alpha_{1}^{\prime}-\alpha_{3}^{\prime} & -\alpha_{13}
\end{array}\right\}_{k_{1}} \\
& \times\left\{\frac{\left(\mu_{1}+1\right) !\left(\mu_{3}+1\right) !}{\left(\lambda_{1}+1\right) !\left(\lambda_{3}+1\right) !}\right\} \\
& \times\left|\overline{\lambda_{1} \mu_{1} ; \alpha_{1}^{\prime}}\right\rangle\left|\overline{\lambda_{3} \mu_{3} ; \alpha_{3}^{\prime}}\right\rangle\left|\lambda_{13} \mu_{13} ; \alpha_{13}\right\rangle .
\end{aligned}
$$

The variables of the four invariants, Eqs. (3.1), have been exchanged such that the functions $h_{i}\left(\rho_{i}\right)$ are still invariants in the triple product space. Further, for each base vector $|\lambda \mu ; \alpha\rangle$, there exists the corresponding base vector $\left|\overline{\lambda \mu ; \alpha^{\prime}}\right\rangle$ with complex conjugate variables. An integral over the product of invariants then yields the inner products, $\left(\left|\lambda \mu ; \alpha^{\prime}\right\rangle\right.$, $|\lambda \mu ; \alpha\rangle)=\delta_{\alpha, \alpha^{\prime}}$, since the base vectors $|\lambda \mu ; \alpha\rangle$ are orthonormal [see Eq. (2.9) of (I)]. If the degree conditions are chosen to give the $3(\lambda \mu)$ coefficients of Eqs. (3.1), then the product of the four $h_{i}\left(\rho_{i}\right)$, integrated over $\zeta_{i}$, should give, within factors $A_{i}=$ $\left[\left(\mu_{i}+1\right) ! /\left(\lambda_{i}+1\right) !\right]^{3}$, the $6(\lambda \mu)$ coefficient on the right-hand side of Eq. (1.1). Thus ${ }^{7}$

$$
\int \prod_{i=1}^{4} h_{j}\left(\rho_{i}\right) d \mu_{36}(\zeta)=C[6(\lambda \mu)]
$$

where

$$
C=\left\{\frac{\left(\lambda_{12}+1\right) !(\mu+1) !\left(\lambda_{2}+1\right) !\left(\mu_{13}+1\right) !\left(\mu_{1}+1\right) !\left(\mu_{3}+1\right) !}{\left(\mu_{12}+1\right) !(\lambda+1) !\left(\mu_{2}+1\right) !\left(\lambda_{13}+1\right) !\left(\lambda_{1}+1\right) !\left(\lambda_{3}+1\right) !}\right\}
$$

The factor $C$ arises because the exchange of variables $\zeta \leftrightarrow \delta$ changes the normalization of the base vector [see Eq. (2.21) of (I)]. As seen in Sec. 3D of (I), it also changes the normalization of the invariant $h_{k}\left(\rho_{i}\right)$ by the same factor. If the four $h_{j}\left(\rho_{i}\right)$ are assumed normalized before the appropriate change in variables, factor $C$ may be dropped. That is, if

$$
\left[h_{1}\left(\zeta_{1}, \delta_{1} ; \zeta_{2}, \delta_{2} ; \zeta_{12}, \delta_{12}\right), h_{1}\left(\zeta_{1}, \delta_{1} ; \zeta_{2}, \cdots\right)\right]=1
$$

The general functional dependence is given by Eqs. (3.33), (3.34), and (3.35) of (I). (and similarly for $h_{2}, h_{3}, h_{4}$ ), then Eq. (3.2) may be written as

$$
\int \prod_{i=1}^{4} h_{i}\left(\rho_{i}\right) d \mu_{36}(\zeta)=[6(\lambda \mu)]
$$

Let $\rho_{i j}$ be the power of the determinants, ${ }^{\circ}$ where $i=1,2,3,4$ labels the particular invariant $h_{i}(\zeta)$, [Eq. (3.1)] and $j=0,1, \cdots, 6,0^{\prime}$ labels the determinant. Let $\kappa_{i j}, i=1, \cdots, 4, j=1, \cdots, 6$, be the partition numbers,

7 The measure $d \mu_{n}(\zeta)$ is defined in Eq. (1.1b) of (I), or see Bargmann (Ref. 5 ). 


$$
\begin{aligned}
& \kappa_{1 i}=\left(\mu_{12}, \lambda_{2}, \lambda_{1}, \lambda_{12}, \mu_{2}, \mu_{1}\right), \\
& \kappa_{2 i}=\left(\mu, \lambda_{3}, \lambda_{12}, \lambda, \mu_{3}, \mu_{12}\right), \\
& \kappa_{3 i}=\left(\lambda, \mu_{2}, \mu_{13}, \mu, \lambda_{2}, \lambda_{13}\right), \\
& \kappa_{4 i}=\left(\lambda_{13}, \mu_{3}, \mu_{1}, \mu_{13}, \lambda_{3}, \lambda_{1}\right) .
\end{aligned}
$$

The degree conditions become

$$
\begin{aligned}
& k_{i 0}+\rho_{i 3}+\rho_{i 6}+N_{i}=\kappa_{i 1}, \\
& k_{i 0}+\rho_{i 1}+\rho_{i 4}+N_{i}=\kappa_{i 2}, \\
& k_{i 0}+\rho_{i 2}+\rho_{i 5}+N_{i}=\kappa_{i 3}, \quad \rho_{i j} \geq 0 \\
& k_{i 0}^{\prime}+\rho_{i 1}+\rho_{i 2}+N_{i}=\kappa_{i 4}, \\
& k_{i 0}^{\prime}+\rho_{i 5}+\rho_{i 6}+N_{i}=\kappa_{i 5}, \\
& k_{i 0}^{\prime}+\rho_{i 3}+\rho_{i 4}+N_{i}=\kappa_{i 6},
\end{aligned}
$$

$P_{i}=\frac{1}{3}\left[\kappa_{i 1}+\kappa_{i 2}+\kappa_{i 3}+2\left(\kappa_{i 4}+\kappa_{i 5}+\kappa_{i 6}\right)\right]$.

The $\rho_{i j}$ of Eq. (3.6) are not independent, e.g., $\mu_{12}$ occurs in Eq. (3.5a) and (3.5b), so that relation $k_{10}+\rho_{13}+\rho_{16}+N_{1}=k_{20}^{\prime}+\rho_{23}+\rho_{24}+N_{2}$ holds. There are 11 other such relations called by Bargmann $^{5}$ the compatibility conditions. Note in the above that either $k_{i 0}$ or $k_{i 0}^{\prime}$ is equal to zero, depending on whether $k_{i 0}-k_{i 0}^{\prime}$ is $\geq 0$ or $<0$, respectively [see Eq. (3.32) of (I)].
If the explicit form of $h_{i}\left(\rho_{i i}\right)$ is inserted in Eq. (3.4), then

$$
[6(\lambda \mu)]=\sum_{n_{i 1}+n_{i 2}=N_{i}} \prod_{m=1}^{4} \beta_{k m}\left(\rho_{m i} ; n_{m 1}, n_{m 2}\right) I\left(k_{i j}\right),
$$

where

$$
\begin{aligned}
& I\left(k_{i j}\right)=\int \prod_{m=1}^{4}\left(H_{m 1}\right)^{n_{m 1}}\left(H_{m 2}\right)^{n_{m 2}} \\
& \times F\left(\rho_{m_{i}}\right) G^{(m)}\left(k_{m 0}, k_{m 0}^{\prime}\right) d \mu_{36}(\zeta)
\end{aligned}
$$

and $G^{(m)}\left(k_{m 0}, k_{m 0}^{\prime}\right)$ represents the determinants raised to the $k_{m 0}$ or $k_{m 0}^{\prime}$ power, e.g., in $h_{1}\left(\rho_{i j}\right)$,

$$
G^{1}\left(k_{10}, 0\right)=\left[\left(\zeta_{1} \times \zeta_{2}\right) \cdot \zeta_{12}\right]^{k_{1} \bullet}, \quad k_{10}-k_{10}^{\prime} \geq 0 \text {. }
$$

To obtain the $6(\lambda \mu)$ coefficients, it would be necessary to integrate Eq. (3.9). This integral may be evaluated, but it would involve numerous sums over a product of factorials. There is no particular utility in presenting it here since, if particular numbers are required, Eqs. (3.8), (3.9), may be programmed. A particularly simple case, the nondegenerate case, is carried out in the next section.

\section{4. $6(\lambda . u)$ SYMBOL FOR THE NONDEGENERATE CASE}

Let $\mu_{1}, \mu_{2}, \mu_{3}=0$. The $6(\lambda \mu)$ symbol becomes

$$
[6(\lambda \mu)]=\left[\begin{array}{ccc}
\lambda \mu & \lambda_{2} 0 & \lambda_{12} \mu_{12} \\
\lambda_{1} 0 & \lambda_{3} 0 & \lambda_{13} \mu_{13}
\end{array}\right] \text {. }
$$

According to Eqs. (3.6), the invariants $h_{i}\left(\rho_{i i}\right)$ are

$$
\begin{aligned}
& h_{1}=\Delta_{1} \frac{\left[\left(\zeta_{1} \times \zeta_{2}\right) \cdot \zeta_{12}\right]^{k_{10}}\left(\zeta_{2} \cdot \delta_{12}\right)^{\rho_{12}}\left(\zeta_{1} \cdot \delta_{12}\right)^{\rho_{13}}}{k_{10} !} \\
& \rho_{11} ! \rho_{12} !
\end{aligned}
$$

where $\Delta_{i}$ is the normalization before the change of variables (the $h_{i}$ above are not normalized to unity). The degree conditions, Eqs. (3.6), become

$$
\begin{aligned}
k_{10} & =\mu_{12}, & & k_{10}+\rho_{11}=\lambda_{2}, \\
k_{10}+\rho_{12} & =\lambda_{1}, & \rho_{11}+\rho_{12} & =\lambda_{12},
\end{aligned}
$$

$k_{20}+\rho_{23}=\mu, \quad k_{20}+\rho_{22}=\lambda_{12}, \quad \rho_{21}+\rho_{22}=\lambda$,

$k_{20}+\rho_{21}+\rho_{24}=\lambda_{3}, \quad \rho_{23}+\rho_{24}=\mu_{12}$,

$\rho_{33}+\rho_{36}=\lambda, \quad \rho_{32}+k_{30}^{\prime}=\mu, \quad \rho_{33}+k_{30}^{\prime}=\lambda_{13}$,

$\rho_{32}+\rho_{35}=\mu_{13}, \quad \rho_{35}+\rho_{36}+k_{30}^{\prime}=\lambda_{2}$,

$$
\begin{gathered}
\rho_{43}+\rho_{46}=\lambda_{13}, \quad k_{40}^{\prime}=\mu_{13}, \\
\rho_{46}+k_{40}^{\prime}=\lambda_{3}, \quad \rho_{43}+k_{40}^{\prime}=\lambda_{1} .
\end{gathered}
$$

Note that since $\lambda_{1}+\lambda_{2}=\lambda_{12}+2 \mu_{12}$ and $\lambda_{1}+\lambda_{3}=$ $\lambda_{13}+2 \mu_{13}$, therefore $\lambda_{12}+2 \mu_{12}+\lambda_{3}=2 \mu_{13}+\lambda_{13}+$ $\lambda_{2}$ and $\rho_{24}=\rho_{35}$.

Divide the invariants $h_{i}$, Eqs. (4.2), by the respective normalizations $\Delta_{i}$,

$$
f_{i}\left(\rho_{i j}\right) \equiv h_{i}\left(\rho_{i i}\right) \cdot\left(\Delta_{i}\right)^{-1} .
$$

Multiply the four $f_{k}\left(\rho_{i j}\right)$ by $\prod_{i i} \tau_{i j}^{p i j}$ and sum over the $\rho_{i i}$, then the following generating function $S\left(\tau_{i i}\right)$ 
is obtained,

$$
\begin{aligned}
S\left(\boldsymbol{\tau}_{i i}\right) & \equiv \sum \frac{[6(\lambda \mu)]}{\Delta_{1} \cdots \Delta_{4}} \Pi \tau_{i j}^{p_{i j}} \\
& =\int \exp \left[\phi\left(\tau_{i j} ; \zeta, \bar{\zeta}\right)\right] d \mu_{27}(\zeta)
\end{aligned}
$$

Conceptually, the remaining steps are clear: integrate where over the variables $\zeta_{1}, \zeta_{2}, \zeta_{12}, \zeta_{12}^{\prime}, \zeta_{3}, \zeta, \zeta^{\prime}, \zeta_{13}, \zeta_{13}^{\prime}$, expand in terms of the parameters $\tau_{i j}$, and the coefficient of this expansion is the $6\left(\lambda_{\mu}\right)$ symbol divided by the normalizations $\Delta_{1} \cdots \Delta_{4}$. This integration is carried out in the Appendix. The result is

$$
[6(\lambda \mu)]=2 C^{\prime} \cdot S\left(N_{12} N_{13}\right)^{-\frac{1}{3}},
$$

$$
\begin{aligned}
C^{\prime} & =\left\{\frac{\left(\lambda_{2}-\mu_{12}\right) !\left(\lambda_{1}-\mu_{12}\right) ! k_{20} ! \rho_{21} ! \rho_{22} ! \rho_{23} ! \rho_{32} ! \rho_{33} ! \rho_{35} ! k_{30}^{\prime} !\left(\lambda_{12}+1\right)\left(\lambda_{13}+1\right)\left(\lambda_{1}-\lambda_{13}\right) !\left(\lambda_{3}-\lambda_{13}\right) !}{\left(P+1-\mu_{12}\right) !\left(P+1-\mu_{13}\right) !\left(\lambda+\mu+\lambda_{13}+\mu_{13}+1-P\right) !\left(\lambda+\mu+\lambda_{12}+\mu_{12}+1-P\right) !}\right\} \\
P & =\frac{1}{3}\left(\lambda_{12}+2 \mu_{12}+\lambda_{3}+2 \lambda+\mu\right)
\end{aligned}
$$

and $S$, in terms of one sum, is

$$
S=\sum \frac{(-1)^{\rho_{14+\lambda_{13}+8}}\left(k_{20}+\rho_{21}+\rho_{22}+\rho_{43}+1-s\right) !}{s !\left(\rho_{12}-s\right) !\left(\rho_{43}-s\right) !\left(\rho_{22}-s\right) !\left[\rho_{11}-\left(\rho_{22}-s\right)\right] !\left(\rho_{32}-s\right) !\left(k_{10}-\rho_{24}-\rho_{43}-s\right) !} .
$$

The general $6(\lambda \mu)$ coefficient has not been evaluated yet, though de Swart ${ }^{1}$ has calculated certain special cases for high-energy physics applications, and $H e c h t^{8.9}$ has the coefficients required for shell model calculations.

\section{APPENDIX}

The method of evaluating Eq. (4.5) is similar to that of $h_{k}\left(\rho_{i}\right)$ given in Appendix B of (I), but the calculation is more laborious. Equation (4.5) is first integrated with respect to $\zeta_{12}, \zeta_{13}, \zeta_{12}^{\prime}, \zeta_{13}^{\prime}$,

$$
S\left(\tau_{i j}\right)=\int \frac{\exp \left[f\left(\zeta, \tau_{i j}\right)\right] d u_{27}}{g\left(\zeta, \tau_{i j}\right)}
$$

where $g\left(\zeta, \tau_{i j}\right), f\left(\zeta, \tau_{i j}\right)$ are functions of the five vectors $\zeta_{1}, \zeta_{2}, \zeta_{3}, \zeta, \zeta^{\prime}$, their complex conjugates, and $\tau_{i j}$. The exponential and the denominator may be

${ }^{8}$ K. T. Hecht, Nucl. Phys. 62, 1 (1965).

- K. T. Hecht, Selected Topics in Nuclear Spectroscopy (North-Holland Publishing Company, Amsterdam, 1964). expanded

$$
S\left(\tau_{i j}\right)=\sum_{m_{i}} c\left(m_{i}\right) I_{1}\left(m_{i}, \tau_{i j}\right),
$$

where $c\left(m_{i}\right)$ are the coefficients of the expansion, and $I_{1}\left(m_{i}, \tau_{i j}\right)$ is an integral over a polynomial function of the above vectors. To calculate $I_{1}\left(m_{i}, \tau_{i j}\right)$, multiply it by a set of parameters $\left.\Pi\left(k_{i}\right)^{m_{1}} / m_{i} !\right)$ and sum over $m_{i}$ so that the integrand may again be put in exponential form:

$$
\begin{aligned}
S_{1}\left(\tau_{i j}\right) & =\sum_{m_{i}} \Pi\left(\frac{\left(k_{i}\right)^{m_{i}}}{m_{i} !}\right) I_{1}\left(m_{i}, \tau_{i i}\right) \\
& =\int \exp \left[h\left(\zeta, \tau_{i j} ; k_{i}\right)\right] d \mu_{15} .
\end{aligned}
$$

This integral may again be evaluated, expanded, and the above process repeated until all integrations have been performed. Finally, reinserting the results into Eq. (A2), the coefficient of the $\tau_{i j}$ 's and the $k_{i}$ 's yield the result, Eq. (4.6). 